\title{
A Fault Feature Extraction Method for a Gearbox with a Composite Gear Train Based on EEMD and Translation-Invariant Multiwavelet Neighbouring Coefficients
}

\author{
Xinyu Pang1,2,* - Baoan Cheng ${ }^{1}$ - Zhaojian Yang ${ }^{1,2}-$ Feng Li $^{1}$ \\ 1 Taiyuan University of Technology, College of Mechanical Engineering, China \\ 2 Shanxi Key Laboratory of Fully Mechanized Coal Mining Equipment, China
}

\begin{abstract}
Although gearboxes with composite gear trains have been widely used in industrial production, it remains difficult to extract their fault signal features due to relatively complex vibration signals. This paper proposes an effective fault feature extraction method based on ensemble empirical mode decomposition (EEMD) and translation-invariant multiwavelet neighbouring coefficients, through which a clear envelope spectrum of gearbox vibration signals can be obtained. Compared with EEMD denoising or translation-invariant multiwavelet denoising using neighbouring coefficients alone, the method combining both of the denoising approaches can not only effectively suppress the signal noise but also fully retain the fault feature information. The presented method was further experimentally verified using a test rig for a gearbox with a composite gear train. Fault diagnosis was conducted with a single fault, such as snaggletooth and abrasion, as well as mixed faults at different locations. The results have shown that this method can effectively extract the fault features and improve the fault detection rate of a gearbox with a composite gear train.

Keywords: composite gear train; feature extraction; EEMD; multiwavelet neighbouring coefficients

Highlights

- $\quad$ This paper proposes a fault feature extraction method based on EEMD and translation-invariant multiwavelet neighbouring coefficients.

- This method can be used for gearboxes with composite gear trains.

- This method was verified in a test rig with a composite gear train.

- $\quad$ The results showed this method is suitable not only for single fault but also for mixed faults.
\end{abstract}

\section{O INTRODUCTION}

A gearbox with a composite gear train is generally composed of an ordinary gear train and a planetary gear train. It has been widely used in the transmission systems of mechanical equipment, such as coal mine shearer's ranging arm gearboxes, wind turbine gearboxes, and automotive transmissions, etc. [1] to [3]. Compared with ordinary gear train [4] to [6], the complex structure of the composite gear train inevitably leads to its complex vibration characteristics. Its vibration signals contain vibration characteristics [7] and [8] of both ordinary gear train and planetary gear train as well as coupled vibration. Therefore, it is of significance to investigate effective fault diagnosis methods for gearboxes with composite gear trains.

However, its fault features are apt to be disturbed by noises, because its vibration signals are weak and mainly in the low-frequency part. As a result, signal denoising is the chief issue that needs to be solved for fault diagnosis [9] and [10]. At present, the time-frequency and multiresolution characteristics of wavelet transform are mainly utilized for signal denoising. A denoising method based on wavelets has been widely applied in engineering, especially wavelet threshold denoising methods [11] to [13].

Given that the wavelet threshold denoising does not consider the relativity of neighbouring wavelet coefficients, Cai and Silverman [14] proposed a denoising method using neighbouring wavelet coefficients (neighbouring coefficients, NeighCoeff), which considers the relativity of neighbouring wavelet coefficients to improve the denoising effect. Through changing the shrinkage factor of neighbouring coefficients to better extract information of vibration characteristics from noises, Yang and Zhao [15] made some improvements to the NeighCoeff denoising method and successfully realized early weak fault diagnosis of bearings. Strela et al. [16] proposed a multiwavelet scalar denoising method by introducing wavelet soft and hard threshold denoising into multiwavelet transform. Downie and Silverman [17] proposed a multiwavelet vector threshold denoising method by taking account of the relativity of the multiwavelet vector coefficients. To eliminate the Gibbs phenomenon, which exists in the above-mentioned multiwavelet threshold denoising 
method, Bui and Cheng [18] applied the translationinvariant denoising method into multiwavelet denoising to demonstrate that a translation-invariant multiwavelet denoising method has excellent denoising characteristics. A denoising method using neighbouring coefficients into multiwavelet denoising, which has achieved good results in electric power systems has been introduced in the literature [19]. Hu et al. [20] applied the translation-invariant multiwavelets denoising method using neighbouring coefficients into fault diagnosis for satellite communication antenna, which has effectively extracted weak characteristics of early faults. Yuan et al. [21] proposed a denoising method based on translation-invariant multiwavelet neighbouring coefficients, which has successfully extracted the fault feature information of early gear cracks influenced by noise.

In addition to the above-mentioned study on the improvement of the denoising method, signal denoising can be combined with empirical mode decomposition (EMD) to further increase the effectiveness of fault feature extraction. Liu et al. [22] combined a threshold denoising method based on EMD with a probabilistic neural network (PNN) to denoise vibration signals of a rotor system and compared it with a wavelet threshold denoising method and a back propagation neural network (BPNN) denoising method. The comparative results showed that the former has better denoising characteristics. Aiming to solve the problem that the diagnosis signals of a planetary gearbox of a wind turbine generator system under variable working condition are obviously nonlinear and nonstationary, which leads to difficulty in fault feature extraction, $\mathrm{Si}$ et al. [23] proposed a new fault feature extraction method based on EMD and demodulated resonance techniques. According to experimental results, this method is feasible in the aspect of the vibration signal denoising and fault feature extraction of a planetary gearbox. Lee et al. [24] proposed a method for bearing test and fault diagnosis based on on ensemble empirical mode decomposition (EEMD), which integrated particle swarm optimization (PSO), Principal Component Analysis (PCA), etc. with EEMD to extract the fault features of bearings. Given the difficulty in extracting multi-fault features of bearings, Gong et al. [25] suggested a fault feature extraction method based on EEMD and spectrum kurtosis (SK), which is applicable to the fault feature extraction of rotor imbalance and multi-faults of bearings. Zhang et al. [26] presented a fault feature extraction method for gear based on EMD, sample entropy, and grey relation to extract the fault features of gears influenced by noise. Experimental results showed that this method could effectively extract the fault feature information of gears. For planetary gear fault diagnosis, Chen et al. [27] proposed a method based on the multi-scale fractal box dimension of complementary ensemble empirical mode decomposition (CEEMD) and extreme learning machine (ELM).

By combining translation-invariant multiwavelet neighbouring coefficients and EEMD, this paper proposes a fault feature extraction method based on EEMD and translation-invariant multiwavelets neighbouring coefficients. The presented method is applied in fault diagnosis of a gearbox with a composite gear train so as to extract the fault features of such a gearbox train, which is disturbed by noise.

The first section of this paper introduces signal feature extraction method based on EEMD and translation-invariant multiwavelet neighbouring coefficients. The second section introduces a test rig for a gearbox with a composite gear train. The experiment and simulation results and discussions are given in sections 3 and 4. Finally, conclusions of the study are provided.

\section{METHODS}

\subsection{EEMD}

EEMD is an improved algorithm of EMD, which is a self-adaptive decomposition method used to process non-linear and non-stationary signals, can decompose the signal into a limited number of intrinsic mode functions (IMF) components ranging from high frequencies to low frequencies. To eliminate mode mixing of EMD, EEMD introduces white Gaussian noise into original signals. By taking advantage of the homogeneous distribution of white Gaussian noise, different scale components of original signals are automatically distributed to an appropriate reference scale. Steps of EEMD decomposition are as follows [28]:

1. Set the signal to be decomposed as $x(t)$, white Gaussian noise with the same length of $x(t)$ as $n_{i}(t)$ to obtain a new signal:

$$
x_{i}(t)=x(t)+n_{i}(t), i=1,2, \ldots, I .
$$

2. Conduct EMD decomposition on noisy signal $x_{i}(t)$ to obtain a new intrinsic mode component $\mathrm{IMF}_{k}^{i}$, in which $k=1,2, \ldots, K$.

3. Repeat step (1) and (2) and add different white Gaussian noise sequence $n_{i}(t)$ each time. 
4. Conduct overall average calculation on each obtained group of $\mathrm{IMF}_{k}^{i}$ components.

$$
\mathrm{IMF}_{k}=\frac{1}{I} \sum_{i=1}^{I} \mathrm{IMF}_{k}^{i}
$$

\subsection{Translation-Invariant Multiwavelets Neighbouring Coefficients}

The principle of traditional multiwavelet threshold denoising is the same as that of single wavelet threshold denoising [29]. Both enable to achieve the goal of denoising by setting a threshold to eliminate partial wavelet coefficients. The former has the advantage of symmetry, compact support, orthogonality and high order vanish moments. Therefore, compared with a single wavelet, it has a better denoising effect. However, this method ignores the relativity of neighbouring wavelet coefficients, which has an impact on the effectiveness of feature information. As a result, the wavelet denoising using neighbouring coefficients proposed by Cai and Silverman [14] is introduced into translation-invariant multiwavelets, which has considered the relativity of multiwavelet coefficients, which can effectively retain the local feature information of signals.

Set $\mathbf{d}_{j, k}^{(h)}$ as the high-frequency coefficient of the $j$ layer with translation amount $h$ after translationinvariant multiwavelet decomposition and define the variable $\theta_{j, k}^{(h)}=\left(\mathbf{d}_{j, k}^{(h)}\right)^{\mathrm{T}}\left(\mathbf{V}_{j}^{(h)}\right)\left(\mathbf{d}_{j, k}^{(h)}\right)$ as a basic variable of multiwavelet denoising using neighbouring coefficients, in which $\mathbf{V}_{j}^{(h)}$ is the covariance matrix of $\mathbf{d}_{j, k}^{(h)}$ and can be estimated using a robust coefficient.

In view of the multiwavelet coefficient and relativity of adjacent points, define a new variable:

$$
\left(S_{j, k}^{(h)}\right)^{2}=\left(\theta_{j, k-1}^{(h)}\right)^{2}+\left(\theta_{j, k}^{(h)}\right)^{2}+\left(\theta_{j, k+1}^{(h)}\right)^{2} .
$$

According to the shrinkage rule of neighbouring coefficients, the coefficient obtained after translationinvariant multiwavelets denoising using neighbouring coefficients reads:

$$
\hat{d}_{j, k}^{(h)}=\left\{\begin{array}{cc}
d_{j, k}^{(h)}\left[1-\left(\frac{\lambda^{(h)}}{S_{j, k}^{(h)}}\right)^{2}\right] & \left(S_{j, k}^{(h)}\right)^{2} \geq\left(\lambda_{j}^{(h)}\right)^{2}, \\
0 & \left(S_{j, k}^{(h)}\right)^{2}<\left(\lambda_{j}^{(h)}\right)^{2}
\end{array}\right.
$$

where, $\lambda_{j}^{(h)}=2 \ln n_{j}^{(h)}$, referring to threshold $n_{j}^{(h)}$ refers to wavelet coefficient of the $j$ layer after multiwavelet decomposition. Obtain signal $x r_{0, k}^{(h)}$ after conducting translation-invariant multiwavelet anti- transform reconstruction denoising on coefficient $\hat{d}_{j, k}^{(h)}$, and then conduct cyclic anti-translation on $x r_{0, k}^{(h)}$ as well as corresponding postprocessing to obtain a set of one-dimensional output signals $y^{(h)}$ and finally conduct the average calculating operation on $y^{(h)}$ to generate a signal after translation-invariant multiwavelets denoising using neighbouring coefficients.

\subsection{Feature Extraction Method Based on EEMD and Translation-Invariant Multiwavelet Neighbouring Coefficients}

Translation-invariant multiwavelet denoising using neighbouring coefficients acts on the entire length of the signal, while noises are generally distributed in the high-frequency part of the signal. To better retain the effective feature information while denoising, the high-frequency part of the signal needs to be denoised and reconstructed with the low-frequency part.

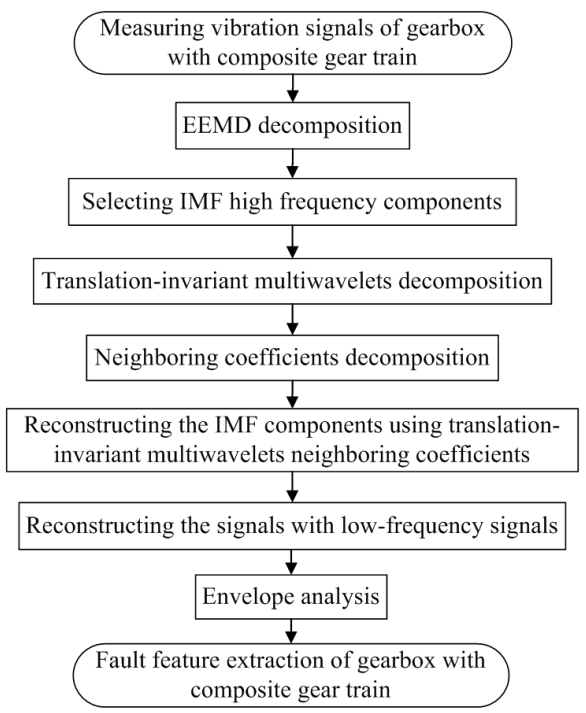

Fig. 1. Procedure of the method

This paper combines the translation-invariant multiwavelet denoising method using neighbouring coefficients with EEMD to diagnose the faults of a gearbox with a composite gear train. The specific procedure of the method is shown in Fig. 1. Firstly, conduct EEMD on the signal to select IMF high-frequency components containing the main information from obtained IMF components according to the energy-correlation coefficient, denoise the IMF high-frequency components with translationinvariant multiwavelet neighbouring coefficients, and then reconstruct them with low-frequency signals to 
obtain the frequency spectrum and spectrum envelope of denoised signal and complete the fault feature extraction of a gearbox with a composite gear train.

\section{EXPERIMENTAL}

\subsection{Test Rig and Fault Gear}

A Drivetrain Diagnostics Simulator (DDS) of SpectraQuest from the US is adapted for the test, as seen in Fig. 2a. The gearbox with a composite gear train is composed of a primary planetary gear and a two-stage spur gear, in which the planetary gearbox has four planet gears with a fixed tooth ring. The schematic diagram of its transmission system is shown in Fig. 2b. The gear parameters are shown in Table. 1. The fault diagnosis test of the gearbox is conducted by pre-setting different gear faults. The test adopts unidirectional acceleration sensors with sample frequency set as $10 \mathrm{kHz}$ and motor speed of 3,000 $\mathrm{r} / \mathrm{min}$.

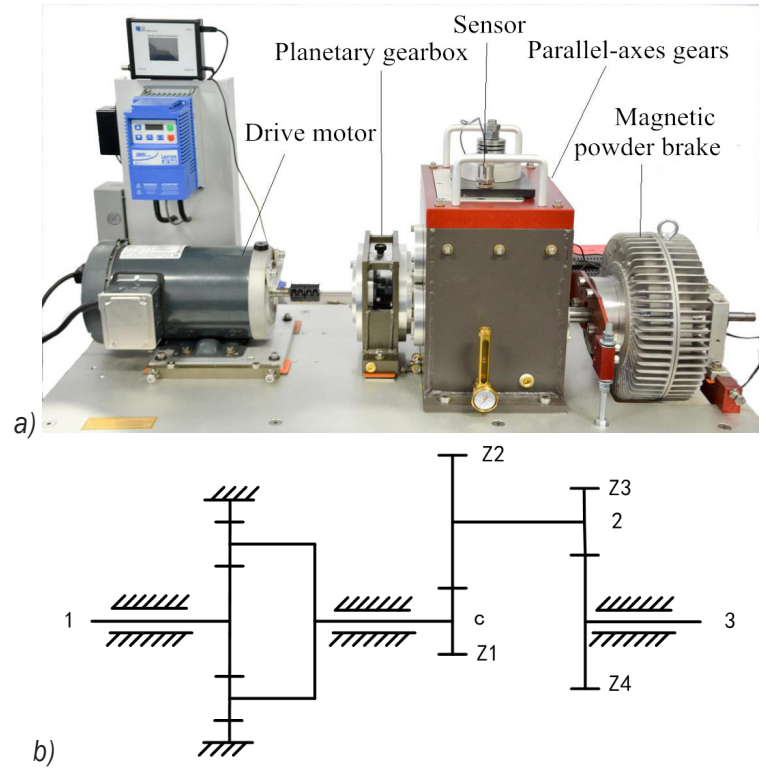

Fig. 2. Test-bed; a) DDS, and

b) transmission structure diagram of gearbox

Table 1. Parameters of gearbox

\begin{tabular}{lclc}
\hline Gear & Teeth number & Gear & Teeth number \\
\hline Sun gear $Z_{s}$ & 28 & Spur Gear $Z_{1}$ & 29 \\
\hline Planet gear $Z_{p}$ & 36 & Spur Gear $Z_{2}$ & 100 \\
\hline Tooth ring $Z_{r}$ & 100 & Spur Gear $Z_{3}$ & 36 \\
\hline & & Spur Gear $Z_{4}$ & 90 \\
\cline { 3 - 4 }
\end{tabular}

According to the distribution location of gear faults, they fall into two categories, i.e. the first category is a localized fault, typically such as snaggletooth, missing tooth, tooth root crack; the other is uniformly distributed faults, typically such as abrasion. The test uses the sun gear and spur gear $Z_{3}$ as fault gears, and the fault types include snaggletooth and abrasion. For the photo of fault gear of gearbox, please refer to Fig. 3.

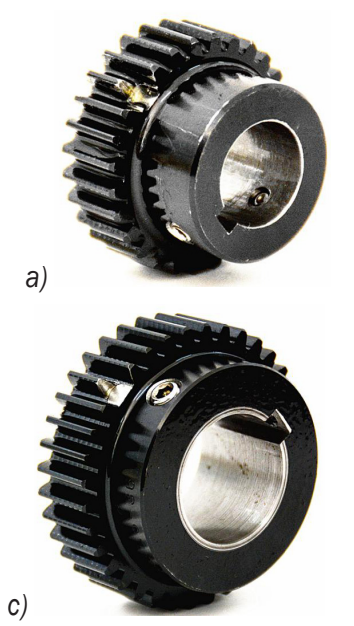

b)

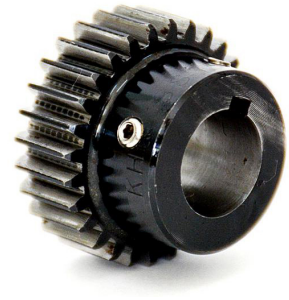

d)

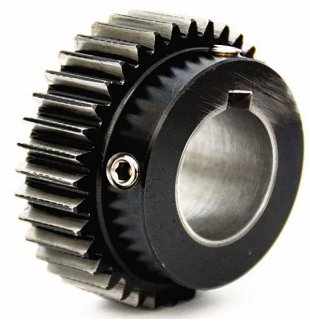

Fig. 3. Fault gear; a) sun gear with snaggletooth, b) sun gear with abrasion, c) spur gear with snaggletooth, and d) spur gear with abrasion

Without loss of generality, the experiment is conducted under three circumstances, i.e. fault-free, single fault and mixed fault. The effect of the feature extraction method based on EEMD and translationinvariant multiwavelet neighbouring coefficients is further verified through a uniformly distributed fault diagnosis test on different types of gearbox faults.

\subsection{Fault Feature Frequency}

The snaggletooth fault frequency of a spur gear is consistent with rotating frequency $f_{c}, f_{\mathrm{s} 2}^{(\mathrm{r})}$ and $f_{\mathrm{s} 3}^{(\mathrm{r})}$ of planet carrier $c$, shaft 2 and shaft 3 . The abrasion fault frequency of the spur gear is consistent with mesh frequency $f_{m 2}$ and $f_{m 3}$ of Z1-Z2 and Z3-Z4. When a fault occurs, the amplitude and frequency multiplication of the rotating frequency or mesh frequency relating to such gear will change accordingly.

Given the revolution and rotation of the planetary gear, its fault frequency is different from that of the spur gear. Generally, the tooth ring is at rest while the sun gear, planetary gear and planet carrier rotate; in such circumstances, the mesh frequency is calculated as:

$$
f_{m}=f_{c} Z_{r}=\left(f_{s}^{(r)}-f_{c}\right) Z_{s},
$$


where $Z_{r}$ refers to tooth number of tooth ring.

The snaggletooth fault frequency of sun gear of the planetary gear train:

$$
f_{s}=N f_{m 1} / Z_{s} .
$$

Abrasion fault frequency of sun gear:

$$
f_{s}=f_{m 1} / Z_{s} .
$$

Snaggletooth fault frequency of planetary gear:

$$
f_{p}=N f_{m 1} / Z_{p} .
$$

Abrasion fault frequency of planetary gear:

$$
f_{p}=f_{m 1} / Z_{p} .
$$

Snaggletooth fault frequency of tooth ring:

$$
f_{r}=N f_{m 1} / Z_{r} .
$$

Abrasion fault frequency of tooth ring:

$$
f_{r}=f_{m 1} / Z_{r} .
$$

\section{EXPERIMENTAL RESULTS AND DISCUSSION}

\subsection{Single Fault}

\subsubsection{Snaggletooth of Spur Gear}

Fig. 4a shows the spectrum envelope of the spur gear's snaggletooth signal. Unlike a normal signal, in addition to the rotating frequency of the planet carrier $f_{c}$, the absolute rotating frequency of the sun gear $f_{s 1}^{(r)}$, and sidebands between both, there also exists a relatively high-frequency peak value, which is the same as rotating frequency of the spur gear, and the snaggletooth of the spur gear can be preliminarily considered. According to the partially amplified lowfrequency part of the spectrum envelope, as shown in Fig. $4 \mathrm{~b}$, in addition to the fault frequency of spur gear $f_{s}$, its frequency multiplication $n f_{s}$ also exists, which proves that the snaggletooth of spur gear occurs in the gearbox with a composite gear train.

\subsubsection{Abrasion of Sun Gear}

Fig. 5 shows the signal spectrum envelope of the sun gear's abrasion fault. It can be seen that the spectrum of the signal is more complex than a normal signal. This is because the abrasion fault is distributed in each tooth of the sun gear; therefore, the former has more frequency components than a normal signal. The signal mainly concentrates around the sun gear's fault frequency $f_{s}$ and the planet gear's fault frequency $f_{p}$.

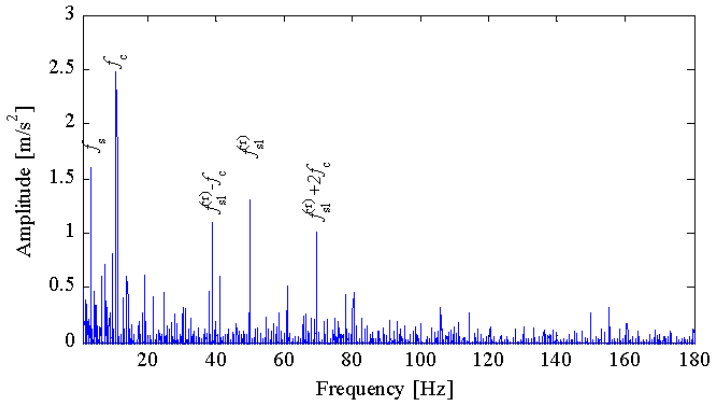

a)

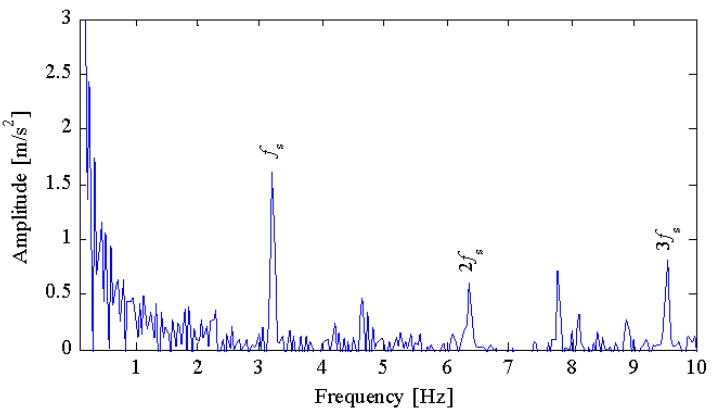

Fig. 4. Signals of spur gear's snaggletooth; a) spectrum envelope, and b) partially amplified low-frequency part

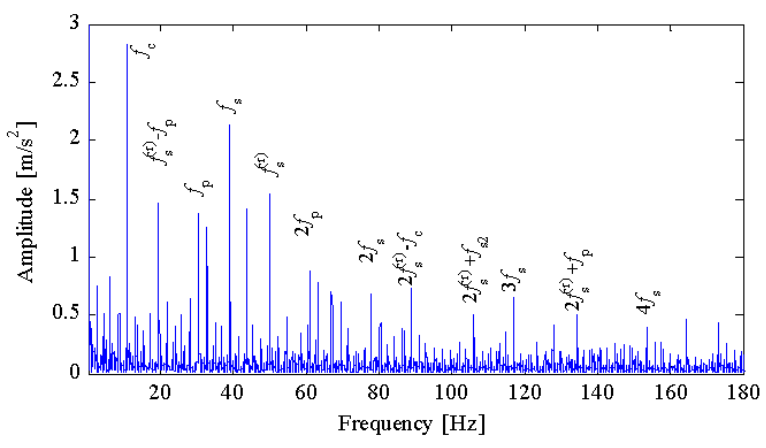

Fig. 5. Signal spectrum envelope of sun gear's abrasion

The main frequencies include the rotating frequency of the planet carrier $f_{c}$, the sun gear's fault frequency $f_{s}$, the planet gear's fault frequency $f_{p}$ and the absolute rotating frequency of the sun gear $f_{s 1}^{(r)}$. Frequency multiplication mainly includes $n f_{p}, n f_{s}$ and sideband components of each signal. Because each tooth of the sun gear is faulty in the sun gear's abrasion fault, the assembly error of planet gear is bigger than that under normal circumstances, and the amplitude of planet gear's fault frequency $f_{p}$ is higher than that under normal state. Furthermore, the sun gear's fault frequency $f_{s}$ and frequency multiplication conform to the frequency feature of the sun gear's abrasion fault, which proves that the sun gear's abrasion occurs in gearboxes with composite gear trains. 


\subsection{Mixed Fault}

In practice, the faults of gearboxes with composite gear trains during operation are not always in the form of a single fault. Instead, multiple faults are often coupled in actual operation. Therefore, multi-fault diagnosis on such gearboxes is also performed. Fig. 6a shows the spectrum envelope of a mixed fault test signal after denoising. The main frequency in the signal includes the rotating frequency of planet carrier $f_{c}$, the absolute rotating frequency of sun gear $f_{s 1}^{(r)}$, frequency $f_{s}$ which is the same as the sun gear's localized fault frequency, the mesh frequency $f_{m 3}$ of spur gear Z3 and Z4. Because the sun gear's snaggletooth fault frequency $f_{s}$ is related to the number of planet gears, and $n / 4$ frequency multiplication such as $1 / 4 f_{s}$ and $2 / 4 f_{s}$ exists in the frequency spectrum, it can be diagnosed that the sun gear snaggletooth fault exists in gearboxes with composite gear trains. In addition, spur gear mesh frequency $f_{m 3}$ is higher than the peak value under the normal state. Therefore, it can be preliminarily ascertained that spur gear mesh frequency-related faults such as abrasion have occurred in gear. In view of this, we zoom in to the frequency range of the spectrum envelope. Fig. $6 \mathrm{~b}$ shows that frequency multiplication such as $2 f_{s}, 2 f_{m 3}$ and $3 f_{m 3}$ also exists in the signal, which proves that mesh frequency $f_{m 3}$ is the fault frequency of spur gear abrasion. In consideration that $f_{m 3}$ is the mesh frequency of spur gear Z3 and Z4 and in order to further determine fault location, the low-frequency part of spectrum envelope is analysed. It is observed that the rotating frequency component of shaft 2 exists in the low-frequency part, which demonstrates that the abrasion fault occurs in spur gear Z3. In conclusion, both the sun gear's snaggletooth fault and the spur gear Z3's abrasion fault occur in gearboxes with composite gear trains, which is consistent with the test results.

According to the results obtained after denoising the vibration signal of gearboxes with composite gear trains under different fault states based on EEMD and translation-invariant multiwavelet neighbouring coefficients and conducting fault diagnosis on the spectrum envelop, this method can effectively improve the signal-to-noise ratio (SNR) of gearboxes with composite gear trains, extract signal feature information flooded in the noise, and help improve accuracy of fault diagnosis for gearboxes with composite gear trains.

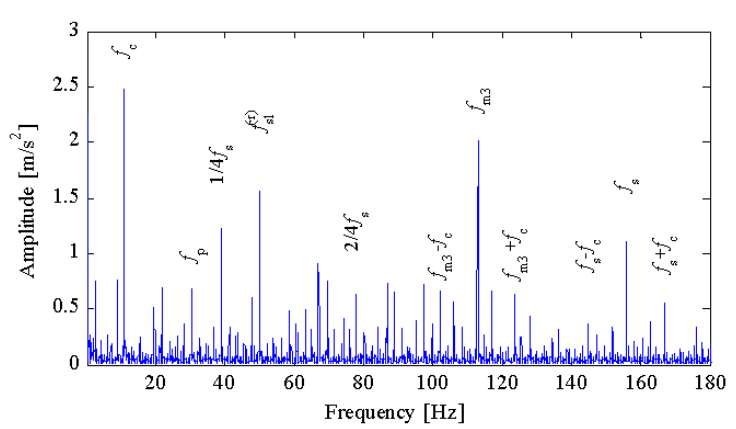

a)

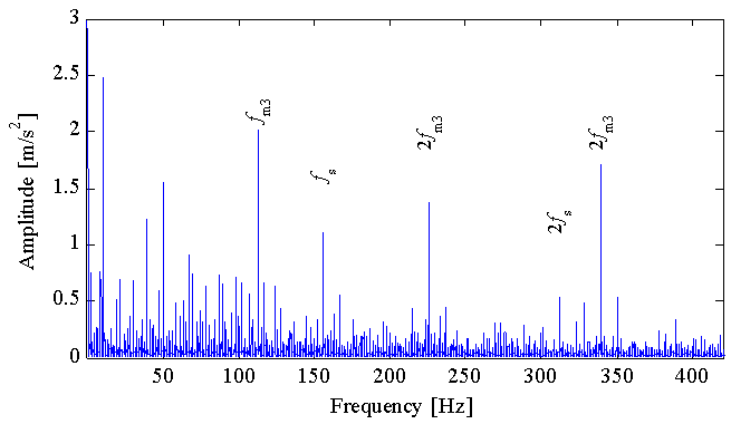

Fig. 6. Spectrum envelope of mixed fault;

a) small-frequency range, and b) large-frequency range

\section{SIMULATION}

\subsection{Simulation Test}

To verify the denoising characteristics of the feature extraction method based on EEMD and the translationinvariant multiwavelet neighbouring coefficients, select a set of modulated signals to simulate the vibration signals of gearboxes with composite gear trains. This model not only includes the modulation characteristics of the gearbox's vibration signals but also simulates the local faults of a group of sun gears of planetary gear trains. The model of vibration signals is shown as follows:

$$
\begin{gathered}
x(t)=x_{1}(t) \cdot\left[1+x_{2}(t)\right]+n(t), \\
x_{1}(t)=\left[1-\cos 2 \pi f_{s 1}^{(r)} t\right]\left[1+A \cos \left(2 \pi f_{s} t+\phi\right)\right] \\
\times \cos \left[2 \pi f_{m 1} t+B \sin \left(2 \pi f_{s} t+\varphi\right)+\theta\right], \\
x_{2}(t)=\cos \left(\pi f_{m 2} t+C \sin 2 \pi f_{s 2}^{(r)} t\right),
\end{gathered}
$$

where, $x_{1}(t)$ refers to the local fault signal of the sun gear, $x_{2}(t)$ refers to the modulated signals of the spur gear, $n(t)$ refers to white Gaussian noise, $A, B$ and $C$ refer to the intensity of amplitude modulation or frequency modulation, respectively, and $\phi, \varphi$ and $\theta$ refer to the initial phases (all set as zero). Those parameters are summarized in Table 2 . The sampling frequency is set as $10 \mathrm{kHz}$. 
Table 2. Simulation signal parameters

\begin{tabular}{lc}
\hline Parameter & Value \\
\hline Intensity of amplitude modulation $A$ & 0.8 \\
\hline Intensity of frequency modulation $B$ & 0.8 \\
\hline Intensity of frequency modulation $C$ & 1.2 \\
\hline Fault frequency $f_{s}$ & $40 \mathrm{~Hz}$ \\
\hline Sun gear rotate frequency $f_{s 1}^{(r)}$ & $15 \mathrm{~Hz}$ \\
\hline Planet gear mesh frequency $f_{m 1}$ & $400 \mathrm{~Hz}$ \\
\hline Spur gear rotate frequency $f_{\mathrm{s} 2}^{(\mathrm{r})}$ & $5 \mathrm{~Hz}$ \\
\hline Spur gear mesh frequency $f_{m 2}$ & $120 \mathrm{~Hz}$ \\
\hline
\end{tabular}

The length of the simulated signals is selected as 4096. The time-domain plot of original signals without noise is seen in Fig. 7a. There is obvious periodic impact in the vibration signals. Fig. $7 \mathrm{~b}$ shows the time-domain plot of original signals added with $-8 \mathrm{~dB}$ white Gaussian noise. Vibration signals of the gearbox are flooded in the noise. Therefore, periodic characteristics cannot be identified.

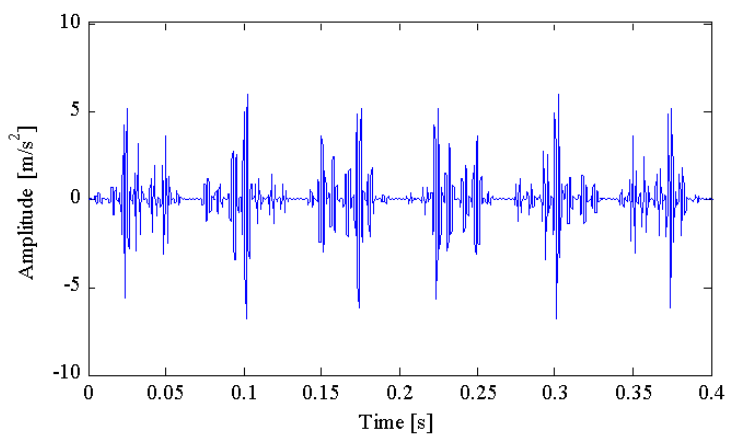

a)

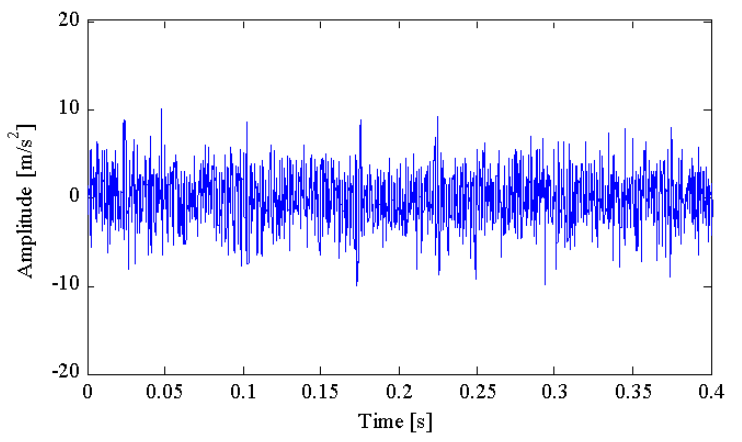

Fig. 7. Original signals; a) without noise, and b) with noise

This paper adopts the most common Geronimo, Hardin, and Massopust (GHM) multiwavelet in multiwavelet signal processing to denoise the noisy signal based on EEMD and translation-invariant multiwavelet neighbouring coefficients, in which the number of multiwavelet decomposition layer is 4 and the cyclic translation amount is 32 .

\subsection{Simulation Result and Analysis}

To highlight the superiority of this method, we conduct denoising on the signal with the other two methods. The first one is EEMD, which achieves denoising by abandoning noisy components; the other one achieves signal denoising through translationinvariant multiwavelets neighbouring coefficients.
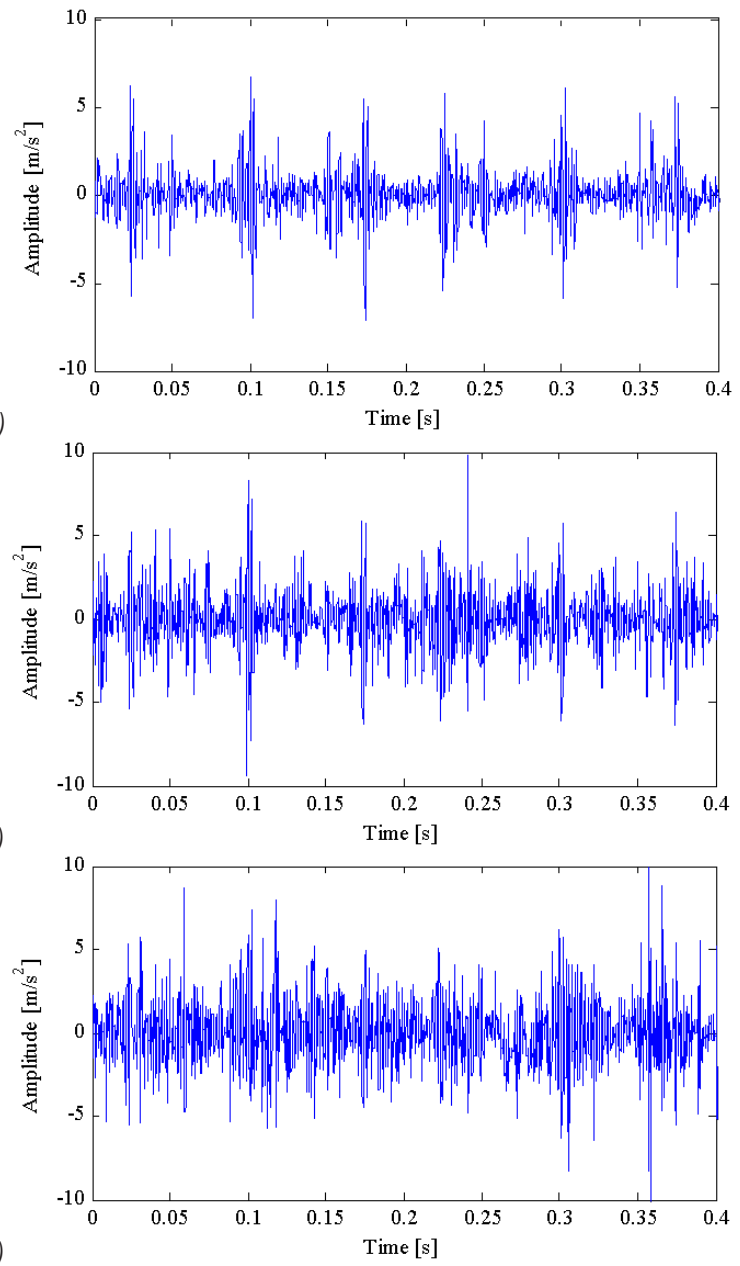

Fig. 8. Time-domain plot of simulated signals denoising with three methods; a) EEMD and translation-invariant multiwavelets neighbouring coefficients, b) EEMD and, c) translation-invariant multiwavelets neighbouring coefficients

Fig. 8 gives the time-domain plot of simulated signals denoising with three methods, respectively. It shows that three methods have obvious denoising effect. For the denoising method based on EEMD and translation-invariant multiwavelets neighbouring coefficients, in Fig. 8a it can be seen that more fully retains shock feature of the signal while achieving denoising. For the time-domain plot of the signal after EEMD decomposition and denoising through 
abandoning partial high-frequency IMF components containing noise, in Fig. $8 \mathrm{~b}$ it is seen that the signal loses partial shock feature while being denoised, which has an impact on the effect of the gearbox's fault diagnosis. Compared with Fig. 8b, for the translation-invariant multiwavelets neighbouring coefficients denoising, as seen in Fig. 8c the signal has less feature information loss, but noise elimination is inadequate and there still exists partial noise feature information in the signal. That could disturb gearbox's fault diagnosis. Corresponding to Fig. 8, the SNR values are respectively 26.59, 19.99, and 16.94, respectively.

The Hilbert envelope analysis on the simulated signal before denoising and the denoised signal obtained through reconstruction with the denoising method based on EEMD and translation-invariant multiwavelets neighbouring coefficients is conducted. Spectrum envelopes of the signals are shown in Fig. 9. Except fault frequency $f_{s}$ and spur gear mesh frequency $f_{m 2}$, the other frequencies in Fig. 9a are disturbed by noise and thus not easily identifiable. After denoising, the signal in Fig. 9b has clear characteristic frequencies, including each main frequency, frequency multiplication and side frequency, which facilitates the fault diagnosis of the gearbox.

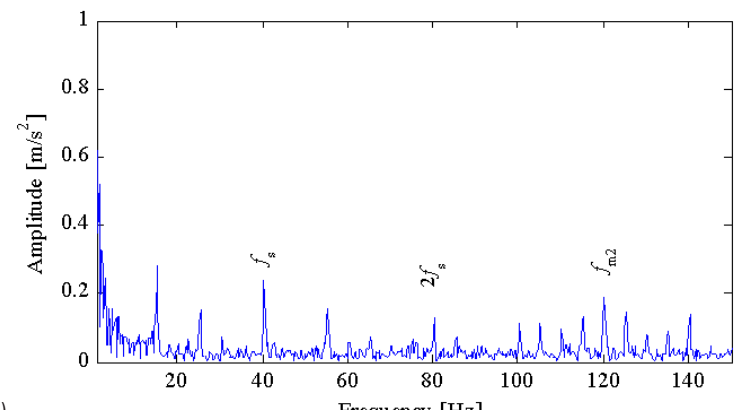

a)

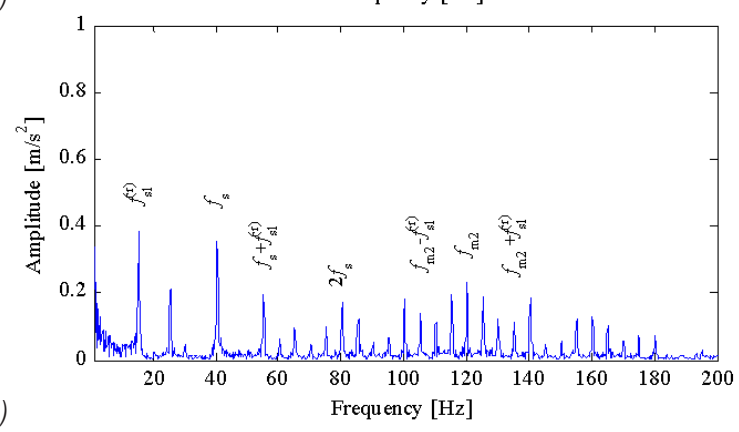

Fig. 9. Envelope spectrum of simulation signal;

a) before denoising, b) after denoising

By comparing the different denoising methods and fault feature extraction and identification, the simulation test proves that the fault diagnosis method of gearboxes with composite gear trains through denoising based on EEMD and translation-invariant multiwavelets neighbouring coefficients cannot only be more effective in improving the SNR of a signal, but also better retain the effective feature information of a signal while achieving denoising. That plays a significant role in improving the fault diagnosis accuracy of a gearbox with a composite gear train.

\section{CONCLUSIONS}

This paper combines EEMD with translation-invariant multiwavelet neighbouring coefficients to denoise the signals of a gearbox with a composite gear train and to extract its signal feature frequency so as to overcome the difficulty in signal feature extraction for such gearboxes. After conducting experimental tests on single fault and mixed faults of the gearboxes with composite gear trains, test results showed that the fault feature extraction method based on EEMD and translation-invariant multiwavelets neighbouring coefficients can effectively extract the fault feature information of such gearboxes, which facilitates the accurate identification of the faults. The simulation test simulates vibration signals containing noise and conducts denoising tests on signals containing noise with different methods. According to the simulation results, the denoising method based on EEMD and translation-invariant multiwavelets neighbouring coefficients cannot only effectively reduce signal noise but also fully retain the effective features of signals.

\section{ACKNOWLEDGEMENTS}

The authors are grateful for the financial support provided by the National Natural Science Foundation of China (51475318 and U1510116).

\section{REFERENCES}

[1] Zhou, D., Zhang, X.F., Zhang, Y.M. (2016). Dynamic reliability analysis for planetary gear system in shearer mechanisms. Mechanism and Machine Theory, vol. 105, p. 244-259, DOI:10.1016/j.mechmachtheory.2016.07.007.

[2] Gong, Y., Fei, J.-L., Tang, J., Yang, Z.-G., Han, Y.-M., Li, X. (2017). Failure analysis on abnormal wear of roller bearings in gearbox for wind turbine. Engineering Failure Analysis, vol. 82, p. 2638, D0I:10.1016/j.engfailanal.2017.08.015.

[3] Kouroussis, G., Dehombreux, P., Verlinden, O. (2015). Vehicle and powertrain dynamics analysis with an automatic gearbox. Mechanism and Machine Theory, vol. 83, p. 109-124, DOI:10.1016/j.mechmachtheory.2014.09.009. 
[4] Li, X.Y., Wang, N.N., Lv, Y.G., Zeng, Q.L., Hidenori, K. (2016). Tooth profile modification and simulation analysis of involute spur gear. International Journal of Simulation Modelling, vol. 15, no. 4, p. 649-662, D0I:10.2507/IJSIMM15(4)6.358.

[5] Zeng, Q.L., Wang, K., Wan, L.R., Zhang, X. (2017). Accurate modelling and transient meshing analysis of involute spur gear based on the principle of gear shaping. International Journal of Simulation Modelling, vol. 16, no. 2, p. 322-333, DOI:10.2507/IJSIMM16(2)C07.

[6] Tang, Z.P., Sun, J.P., Yan, L., Zou, F. (2017). Dynamic contact analysis and tooth modification design for EMU traction gear. International Journal of Simulation Modelling, vol. 16, no. 4, p. 742-753, DOI:10.2507/IJSIMM16(4)C020.

[7] Sharmaa, V., Parey, A. (2016). A review of gear fault diagnosis using various condition indicators. Procedia Engineering, vol. 144, p. 253-263, D0I:10.1016/j.proeng.2016.05.131.

[8] Praveenkumara, T., Saimuruganb, M., Krishnakumarc, P., Ramachandran, K.I. (2014). Fault diagnosis of automobile gearbox based on machine learning techniques. Procedia Engineering, 12th Global congress on manufacturing and management, vol. 97, p. 2092-2098, DOI:10.1016/j. proeng.2014.12.452.

[9] Moshrefzadeh , A., Fasana, A. (2017). Planetary gearbox with localised bearings and gears faults: simulation and time/ frequency analysis. Meccanica, vol. 52, no. 15, p. 3759-3779, D0I:10.1007/s11012-017-0680-7.

[10] Merainani, B., Rahmoune, C., Benazzouz, D., OuldBouamama, B. (2018). A novel gearbox fault feature extraction and classification using Hilbert empirical wavelet transform, singular value decomposition, and SOM neural network. Journal of Vibration and Control, vol. 24, no. 12, p. 25122531, DOI:10.1177/1077546316688991.

[11] Deak, K., Mankovits, T., Kocsis, I. (2016). Optimal wavelet selection for the size estimation of manufacturing defects of tapered roller bearings with vibration measurement using Shannon Entropy Criteria. Strojniški vestnik - Journal of Mechanical Engineering, vol. 63, no. 1, p. 3-14, Dol:10.5545/ sv-jme.2016.3989.

[12] Kumar, R., Singh, M. (2013). Outer race defect width measurement in taper roller bearing using discrete wavelet transform of vibration signal. Measurement, vol. 46, no. 1, p. 537-545, D0I:10.1016/j.measurement.2012.08.012.

[13] Lu, J.-Y., Lin, H., Dong, Y., Zhang Y.-S. (2016). A new wavelet threshold function and denoising application. Mathematical Problems in Engineering, p. 1-8, D0I:10.1155/2016/3195492.

[14] Cai, T.T., Silverman, B.W. (2001). Incorporating information on neighbouring coefficients into wavelet estimation. Sankhya: The Indian Journal od Statistic: Series B, vol. 63, no. 2, p. 127 148.

[15] Yang, S.P., Zhao. Z.H. (2013). Improved wavelet denoising using neighboring coefficients and its application to machinery fault diagnosis. Journal of Mechanical Engineering, vol. 49, no. 17, p. 137-141, Dol:10.3901/JME.2013.17.137.

[16] Strela, V., Heller, P.N., Strang, G. Topiwala, P., Heil, C. (1999). Application of multiwavelet filterbanks to image processing. IEEE Transactions on Image Processing, vol. 8, no. 4 p. 548563, DOI:10.1109/83.753742.
[17] Downie, T.R., Silverman, B.W. (1998).Discrete multiple wavelet transform and thresholding methods. IEEE Transactions on Signal Processing, vol. 46, no. 9, p. 2558-2561, DOl:10.1109/78.709546.

[18] Bui, T.D. Chen, G.Y. (2002). Translation-invariant denoising using multiwavelets. IEEE Transactions on Signal Processing, vol. 46, no. 12, p. 3414-3420, Dol:10.1109/78.735315.

[19] Chen, G.Y., Bui, T.D. (2003). Multiwavelets denoising using neighboring coefficients. IEEE Signal Processing Letters, vol. 10, no. 7, p. 211-214, D0I:10.1109/LSP.2003.811586.

[20] Hu, X., Xi, Z., Li, K., Cong, B. (2013). Application of translationinvariant multiwavelets denoising using neighboring coefficients to fault diagnosis of antennas on vessel. Proceedings International Conference on Mechatronic Sciences, Electric Engineering and Computer, p. 870-873, DOI:10.1109/MEC.2013.6885181.

[21] Yuan, J., He, Z.J., Wang, X.D., Li, Z., Zi, Y.Y. (2009). Translationinvariant multiwavelets denoising using neighboring coefficients and its application to monitoring and diagnosis. Journal of Mechanical Engineering, vol. 45, no. 4, p. 155-160, DOI:10.3901/JME.2009.04.155.

[22] Liu, D., Zeng, H.T. Xiao, Z.H., Peng, L.H., Malik,O.P. (2017). Fault diagnosis of rotor using EMD thresholding-based denoising combined with probabilistic neural network. Journal of Vibroengineering, vol. 19, no. 8, p. 5920-5931, Dol:10.21595/ jve.2017.18365.

[23] Si, J.S., Cao, Y., Shi, X.J. (2017). Fault diagnosis of wind turbine planetary gear box based on EMD and resonance remodulation. 12 $12^{\text {th }}$ International Conference on Computer Science and Education, p. 757-762, D0l:10.1109/ ICCSE.2017.8085595.

[24] Lee, D.H., Ahn, J.H., Koh, B.H. (2017). Fault Detection of Bearing Systems through EEMD and Optimization Algorithm. Sensors, vol. 17, no. 11, p. 2477, D0l:10.3390/s17112477.

[25] Gong, X.Y., Du, W.L., Georgiadis, A., Zhao, B.W. (2017). Identification of multi-fault in rotor-bearing system using spectral kurtosis and EEMD. Journal of Vibroengineering, vol. 19, no. 7, p. 5036-5046, Dol:10.21595/jve.2017.18671.

[26] Zhang, W.B., Pu, Y.S., Zhu, J.X., Su, Y.P. (2013). Gear Fault Diagnosis Method Using EEMD Sample Entropy and Grey Incidence. Advanced Materials Research, vol. 694-697, p. 1151-1154, D0l:10.4028/www.scientific.net/AMR.694697.1151.

[27] Chen, X.H., Cheng, G., Li, H.Y., Li, Y. (2017). Research of planetary gear fault diagnosis based on multi-scale fractal box dimension of CEEMD and ELM. Strojniški vestnik Journal of Mechanical Engineering, vol. 63, no. 1, p. 45-55, DOl:10.5545/sv-jme.2016.3811.

[28] Leng, J.F., Jing, S.X. Luo, C.X., Wang, Z.Y. (2017). EEMD-Based cICA method for single-channel signal separation and fault feature extraction of gearbox. Journal of Vibroengineering, vol. 19, no. 8, p. 5858-5873, Dol:10.21595/jve.2017.18115.

[29] Hua, W., Niu, Z.H., Wang, Z.Y., Leng,J.F. (2016). Mine gearbox fault diagnosis based on neighboring coefficients of translation-invariant multiwavelets. Journal of China Coal Society, vol. 41, no. s1, p. 253-258, D0l:10.13225/j.cnki. jccs.2015.0968. 